Int. J. Dev. Biol. 56: 165-171

doi: $10.1387 / \mathrm{ijdb} .113433 \mathrm{hn}$

\title{
The Dr-nanos gene is essential for germ cell specification in the planarian Dugesia ryukyuensis
}

\author{
HARUKA NAKAGAWA ${ }^{1,2}$, HIROTSUGU ISHIZU ${ }^{1,2}$, AYAKO CHINONE, \\ KAZUYA KOBAYASHI ${ }^{1}$ and MIDORI MATSUMOTO* \\ Department of Biological Sciences and Informatics, Keio University, Hiyoshi, Kouhoku-ku, Yokohama, Japan
}

\begin{abstract}
Homologs of nanos are required for the formation and maintenance of germline stem cell (GSC) systems and for gametogenesis in many metazoans. Planarians can change their reproductive mode seasonally, alternating between asexual and sexual reproduction; they develop and maintain their somatic stem cells (SSCs) and GCSs from pluripotent stem cells known as neoblasts. We isolated a nanos homolog, Dr-nanos, from the expressed sequence tags (ESTs) of the sexualized form of Dugesia ryukyuensis. We examined the expression of Dr-nanos in asexual and sexualized planarians by in situ hybridization and analyzed its function using RNA interference (RNAi) together with a planarian sexualization assay. A nanos homolog, Dr-nanos, was identified in the planarian D. ryukyuensis. Dr-nanos expression was observed in the ovarian primordial cells of the asexual worms. This expression increased in proportion to sexualization and was localized in the early germline cells of the ovaries and testes. In X-ray-irradiated worms, the expression of Dr-nanos decreased to a large extent, indicating that Dr-nanos is expressed in some subpopulations of stem cells, especially in GSCs. During the sexualization process, worms in which Dr-nanos was knocked down by RNAi exhibited decreased numbers of oogonia in the ovaries and failed to develop testes, whereas the somatic sexual organs were not affected. We conclude that Dr-nanos is essential for the development of germ cells in the ovaries and testes and may have a function in the early stages of germ cell specification, but not in the development of somatic sexual organs.
\end{abstract}

KEY WORDS: planarian, Nanos, germline stem cell, germ cell specification

\section{Introduction}

Germ cells are specialized cells that transmit genetic information from generation to generation during sexual reproduction. They are totipotent stem cells that can differentiate into any cell type. Germ cells must form correctly in order to produce offspring and to ensure the survival of the species through the generations. There are at least 2 distinct modes of germline segregation in metazoans: germ cells can be specified by maternally inherited determinants (preformation) and by inductive signals (epigenesis) (Extavour and Akam, 2003; Johnson et al., 2003).

The nanos gene encodes an RNA-binding protein containing a zinc finger motif, which is conserved in diverse metazoans. In Drosophila, nanos is required for abdominal segmentation (Lehmann and Nüsslein-Volhard, 1991; Wang and Lehmann, 1991) and germ cell differentiation and maintenance (Forbes and Lehmann, 1991; Kobayashi et al., 1996; Hayashi et al., 2004). In nematoda, zebrafish, and mice, orthologs of nanos are reported to be involved in the migration of the primordial germ cells (PGCs) to the gonads, and in the transcriptional quiescence and mitotic arrest required for the maintenance of germline identity during migration (Subramaniam and Seydoux, 1999; Köprunner et al., 2001; Tsuda et al., 2003; Parisi and Lin, 2000; Suzuki et al., 2007).

Freshwater planarians exhibit a robust regenerative ability

Abbreviations used in this paper: dsRNA, double strand RNA; EST, expressed sequence
tag; GSC, germline stem cell; HEPES, 4-(2-hydroxyethyl)-1-piperazineethanesul-
fonic acid)); PGCs, primordial germ cells; RACE, 3' and 5' rapid amplification of
cDNA ends; RNAi, RNA interference; RT-PCR, reverse transcription polymerase
chain reaction; SSC, somatic stem cell; WISH, whole-mount in situ hybridization.

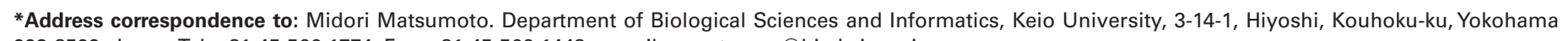
223-8522, Japan. Tel: +81-45-566-1774. Fax: +81-45-566-1448. e-mail: mmatsumo@ bio.keio.ac.jp

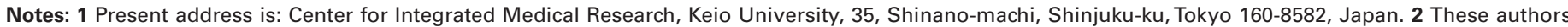
have contributed equally to this work.

Final, author-corrected PDF published online: 16 March 2012

ISSN: Online 1696-3547, Print 0214-6282

(C) 2012 UBC Press

Printed in Spain 

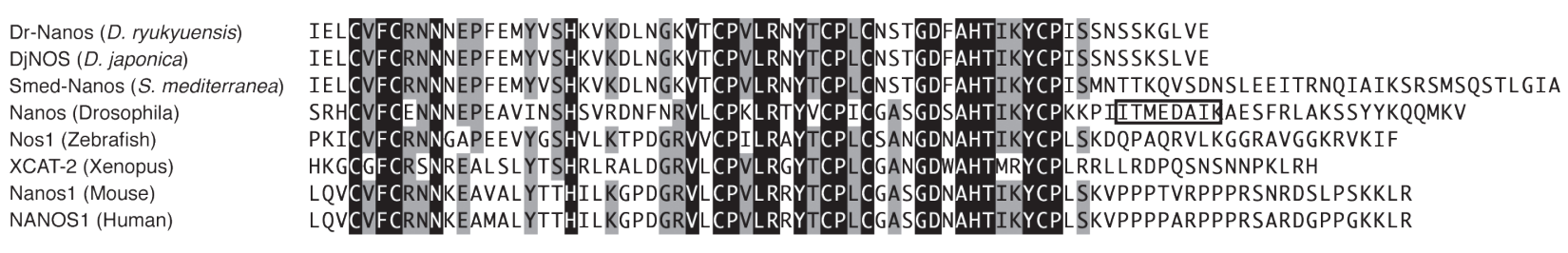

Fig. 1. Alignment of the C-terminal region of the NANOS proteins from different species. NANOS zinc-finger domain is indicated by the black line; black boxes indicate identical residues in all aligned proteins; gray boxes indicate residues conserved in at least 5 of the aligned proteins; open boxes indicate the tail end region required for germ cell migration. The full-length cDNA and protein sequences of Dr-Nanos have been deposited in DDBJ/ EMBL/GenBank databases (http://www.ddbj.nig.ac.jp) with the accession number AB650592.

that is mediated by the neoblasts - a type of pluripotent stem cells (Wolff and Dubois, 1948; Baguñà et al., 1989). Many planarians can be categorized according to their different reproductive modes: exclusively asexual, seasonally sexual, and/or exclusively sexual (Jenkins, 1967). Although sexual planarians do not reproduce asexually or by spontaneous fission, they can regenerate all their tissues, including the germline cells and somatic cells of the reproductive organs, after artificial amputation (Morgan, 1901; Newmark et al., 2008). Therefore, neoblasts apparently give rise to both somatic stem cells (SSC) and germline stem cells (GSCs). The GSCs are essential for the continuous production of gametes and are present in a variety of animals ranging from Hydra to insects and mammals (Lin, 1997;1998). Reproducing in one of the modes described above by the influence of environmental conditions facilitates survival of the species. It is very important that a certain amount of GSCs are maintained during asexual reproduction in order to initiate the immediate formation of the germ cells following the switch to the sexual reproductive mode.

Recent studies of nanos in the planarians Dugesia japonica and Schmidtea mediterranea suggested that nanos-positive cells in asexual worms are GSCs, and in sexual worms, they are the oogonia and spermatogonia; the germ cells are differentiated from GSCs according to the development of the reproductive organs (Sato et al., 2006; Wang et al., 2007; Handberg-Thorsager and Saló, 2007). However, the function of the nanos gene during the change from asexual to sexual reproduction remains unknown.

To investigate the functions of the nanos gene during the conversion of the reproductive modes, we have established an experimental bioassay system to induce sexual switching (sexualization) (Kobayashi et al., 1999). In this system, the $\mathrm{OH}$ strain of $D$. ryukyuensis, which has been maintained through asexual reproduction without gonads, can be induced to reproduce sexu- ally by feeding worms of an exclusively oviparous species. During sexualization, the germline cells and the somatic cells of the reproductive organs, such as the ovaries, testes, genital pores, and yolk glands are differentiated in the same order and in similar positions during the developmental process of sexual individuals. This experimental system of sexualization enables us to follow the development of the germline cells in detail, and it is possible to evaluate the function of nanos during germ cell formation by a combination of RNA interference (RNAi) and this assay system. In this study, we isolated the Dr-nanos gene and characterized its function in germline cells and in reproductive organ formation.

\section{Results}

\section{Identification of the Dr-nanos gene}

The nanos homolog was isolated from the expressed sequence tags (ESTs) of the sexualized form of $D$. ryukyuensis and was named Dr-nanos. Dr-nanos encodes a protein of 179 amino acids, with 2 conserved NANOS zinc finger motifs (Fig. 1). Dr-Nanos showed a high sequence identity with NANOS of $D$. japonica (DjNOS; $97.8 \%$ ) and S. mediterranea (Smed-Nanos; 80.4\%). However, the $\mathrm{C}$-terminus domain, following the zinc finger motifs seen in the insects, did not exist in NANOS of the planarians.

\section{Expression patterns of Dr-nanos in the asexual planarian}

The nanos-positive cells observed in the asexual planarian worms were presumed to be the GSCs. To examine the expression pattern of Dr-nanos, we performed whole-mount in situ hybridization (WISH) in the asexual D. ryukyuensis $\mathrm{OH}$ strain. Dr-nanos was expressed in the cells clustered along 2 ventral lines (Fig. 2A). In the anteroventral mesenchyme of the asexual worm, oogonialike cells form small cell clusters known as the ovarian primordia
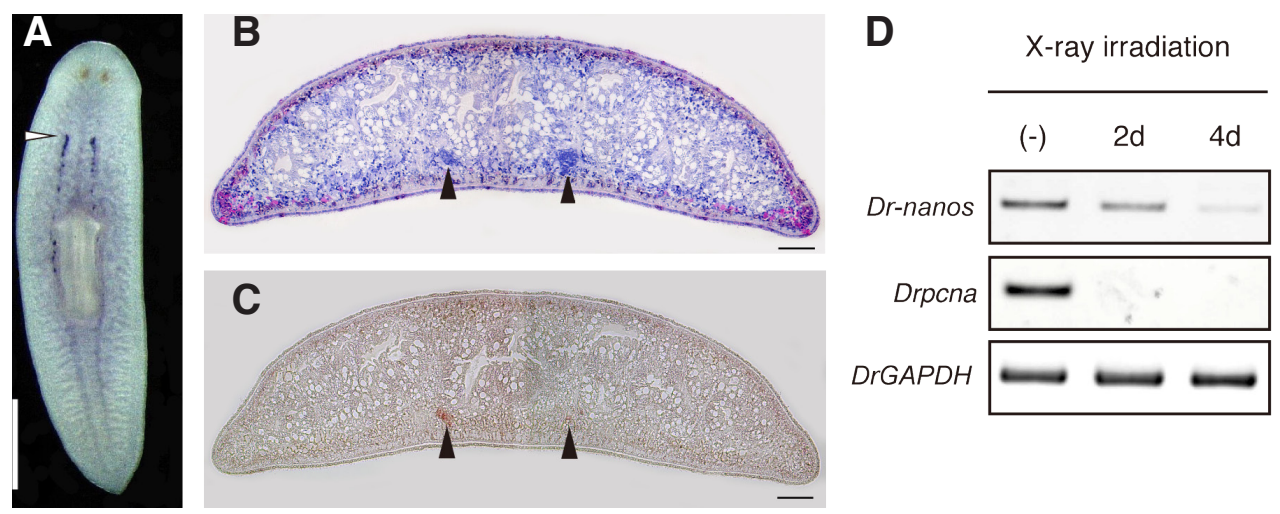

Fig. 2. Analysis of Dr-nanos expression in the asexual planarian $D$. ryukyuensis. (A) Whole-mount in situ hybridization (WISH) in an intact planarian. Dr-nanos expression observed in the anteroventral region-the presumptive ovarian region-indicated by white arrowheads. Scale bar: $1 \mathrm{~mm}$. (B,C) Transverse section of the region of the ovarian primordia: (B) HE stain and (C) in situ hybridization. Dr-nanos expression in the ovarian primordia is indicated by black arrowheads. Scale bars, 100 um. (D) Expression levels of the Dr-nanos, Drpcna, and GAPDH genes after X-ray irradiation, examined by RT-PCR. (-), Not irradiated; 2d, 2 days after irradiation; 4d, 4 days after irradiation. 
A

\section{Sexualization (weeks)}

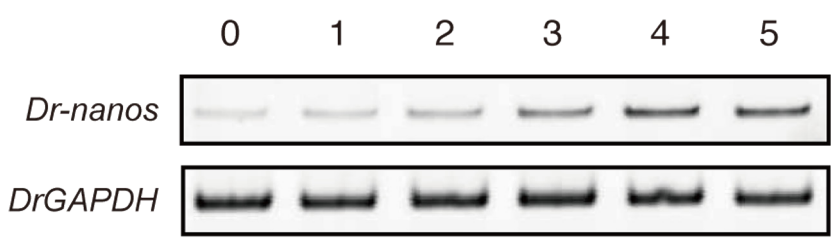

B

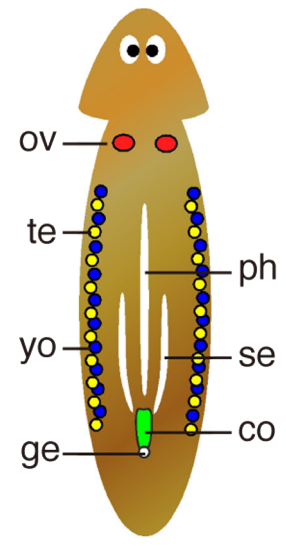

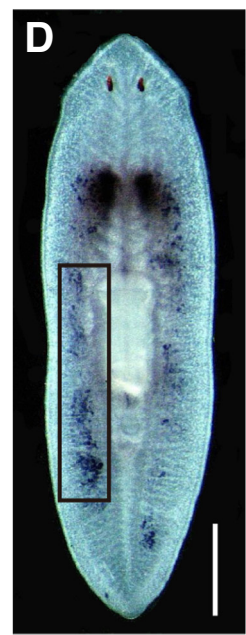

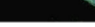

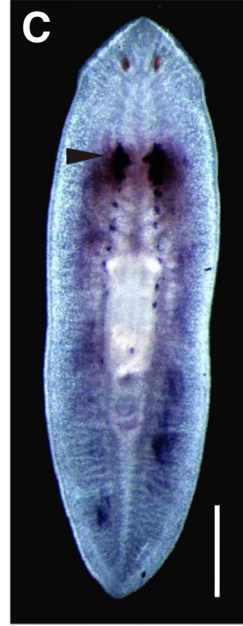

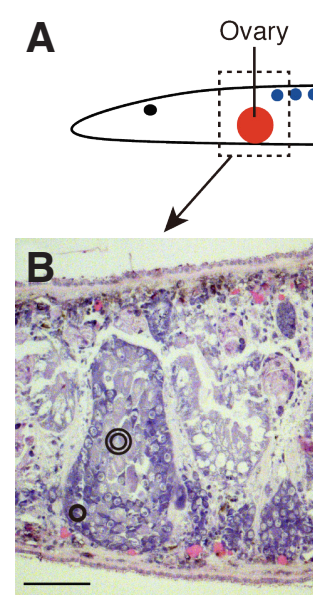

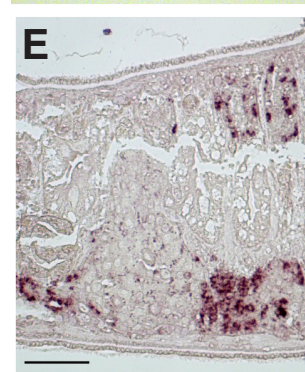

Fig. 3 (Left). Analysis of Dr-nanos gene expression in sexualized worms. (A) Comparison of Dr-nanos expression levels during sexualization, examined by RT-PCR. (B) Scheme of a sexualized planarian: ph, pharynx; ov, ovary; te, testis; yo, yolk gland; se, seminal vesicle; co, copulatory apparatus; and ge, genital pore. (C,D) Dr-nanos expression in sexualized worms detected by WISH: (C) ventral views; (D) dorsal views. Expression in ovaries is indicated by arrowheads. The black box indicates expression in the testes. Scale bars, $1 \mathrm{~mm}$.

(Fig. 2B). Then, using in situ hybridization on sections from these cells, Dr-nanos signals were specifically detected in the cells of the ovarian primordia (Fig. 2C). In many cases, sexualized worms developed several supernumerary ovary pairs along the ventral nerve cords, which reached the pharyngeal level (Sakurai, 1981; Kobayashi and Hoshi, 2011). The cells indicated by WISH may be in the presumed ovarian region. Meanwhile no such cell clusters were observed in the dorsal region, which is the presumed testicular region, and the Dr-nanos signal was not detected in this region.

It is well known that X-ray irradiation causes the loss of regenerative ability by the rapid and specific destruction of the neoblasts (Wolff and Dubois, 1948; Baguñà et al., 1989; Orii et al., 2005). To compare the characteristics of the neoblasts and Dr-nanos-positive cells, we examined changes in Dr-nanosmRNA levels 2 and 4 days after irradiation. Two days after X-ray irradiation, elimination of the neoblasts was confirmed, because Drpcna, which is necessary for DNA replication, was almost eliminated. The mRNA levels of Dr-nanos were largely decreased after 2 days of $\mathrm{X}$-ray irradiation, and decreased further after 4 days (Fig. 2D).

\section{Expression patterns of Dr-nanos in germline cells}

To examine the expression pattern of Dr-nanos in germline cells, the change in Dr-nanos mRNA levels was examined by comparative RT-PCR during the process of sexualization. The expression of Drnanos increased during sexualization (Fig. 3A).

WISH of the sexualized worms revealed that $D r$-nanoswas expressed in the ovaries in the anteroventral area and in the testes in the bilateral dorsal area (Fig. 3 C,D). In situ hybridization observed on sections indicated that Dr-nanos mRNAs were expressed in the ovaries and testes. In the early stages of planarian gonad development, the oocytes are located in the center of the ovary and the oogonia are located in the ventral periphery. The most peripheral oogonium-like cells outside an ovarian mass may be early oogonia or proliferated germline stem cells (Fig. 4B, C). Similarly, in the testes, the spermatogonia and spermatocytes are observed in the periphery, and the spermatid and sperm are located in the center of the testes (Fig. 4D). Dr-nanos was mainly expressed in the more peripheral cells

Fig. 4. Analysis of Dr-nanos expression in germline cells. (A) Scheme of a sagittal section of a sexualized planarian. The dashed boxes indicate the ovarian region (B) and testicular region (D). (B-D) HE stain (E-G) In situ hybridization of a sagittal section for Dr-nanos. (B, E) Region around the ovaries; (C, F) higher magnification in the peripheral region of ovaries. The dashed lines separate each ovary. $(D, G)$ Testes Closed circle, early oogonia; open circles, oogonia; double circle, oocytes; arrow, spermatogonia; asterisks, spermatocyte. Scale bars, $100 \mu \mathrm{m}$. 

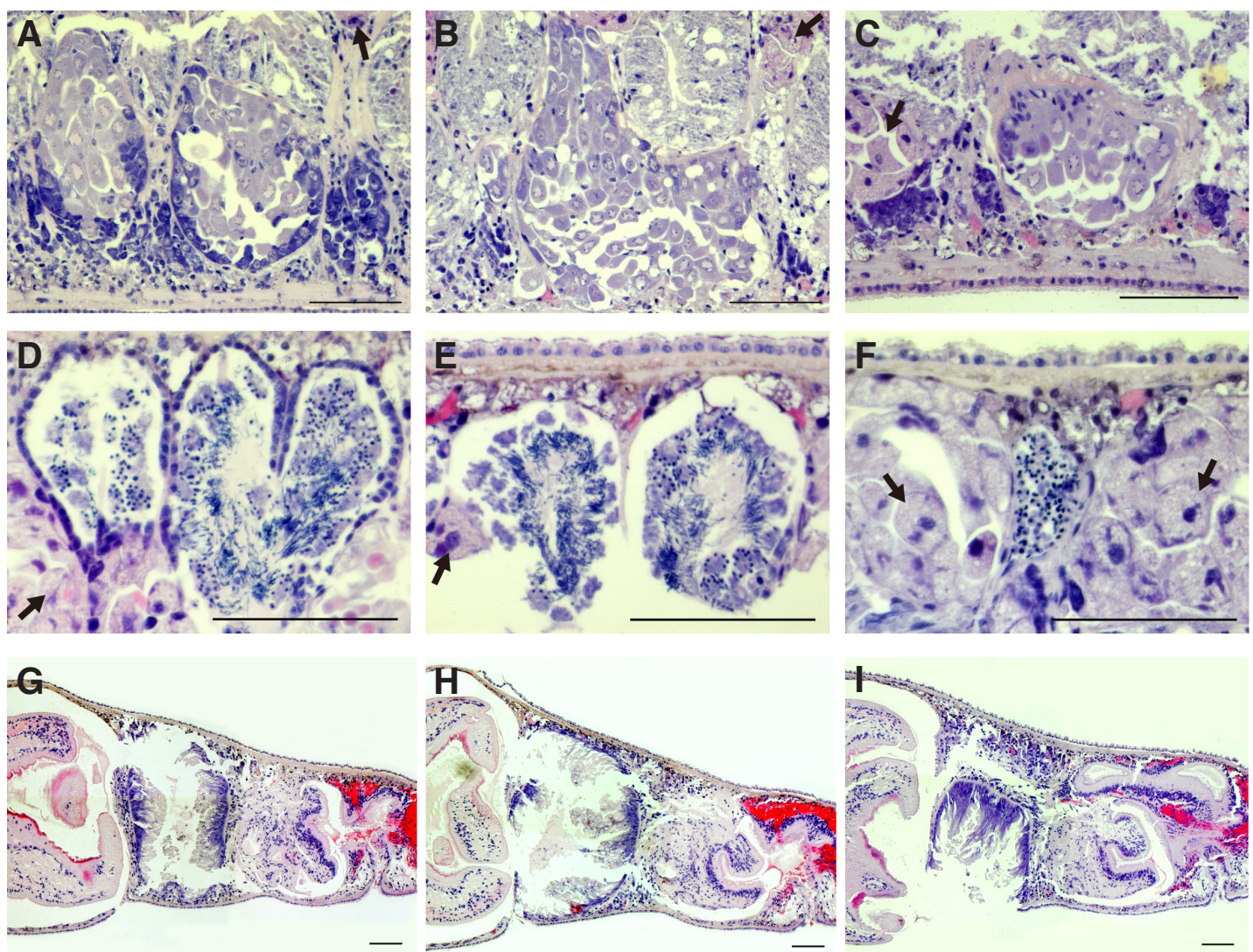

Fig. 5. Analysis of Dr-nanos function during maintenance of sexual worms. (A-F) HE staining of sagittal sections of the acquired sexual worms with Dr-nanos RNAi. (A-C) Ovaries, (D-F) testes, and (G-I) copulatory apparatus. (A, D, and G) Day 0 with dsRNA feeding; (B) ovary at 20 days and (C) 40 days; (E) testes at 20 days and (F) 30 days; (H) copulatory apparatus at 20 days and (I) 50 days. Arrows indicate yolk glands. Scale bars, 100 um.

of the ovaries, i.e., the early oogonia or GSCs. Its expression was weaker in the oogonia than in the GSCs, and it was not detected at all in the oocytes (Fig. 4 E,F). In the testes, Dr-nanos was expressed as expected in the spermatogonia; however, expression signals were not detected in the spermatids or in the sperm inside the testes (Fig. 4G).

\section{Functional analysis of Dr-nanos during the maintenance of sexual worms}

To identify which process of germ cell formation is regulated by Dr-nanos, we performed an RNAiknockdown on Dr-nanosin mature sexualized worms with fully developed reproductive organs and germ cells. Twenty days after Dr-nanos silencing, spermatids and sperm were observed inside the testes, but no spermatogonia or spermatocytes were detected in the periphery (Fig. 5E). Similarly, the ovaries contained oocytes but no oogonia (Fig. 5B). Despite this, the copulatory apparatus (Fig. $5 \mathrm{H}$ ) and the yolk glands (Fig. $5 \mathrm{~B}, \mathrm{E})$ had developed normally with the correct morphology. The testes became smaller at 30 days (Fig. 5F) and had completely disappeared at 40 days. The ovaries were not changed at 30 days, became extremely small at 40 days (Fig. 5C), and finally, were hardly visible at 50 days. The copulatory apparatus (Fig. 5I) and the yolk glands (Fig. 5 C,F) did not regress at 50 days.

\section{Functional analysis of Dr-nanos during germ cell formation}

To investigate the role of Dr-nanos in the process of germ cell formation from germline precursor, we performed RNAi on Dr-nanos with the sexualization assay system. In the Dr-nanos(RNAi) worms, the testes did not develop, and no primordial or immature testes were observed (Fig. 6B). The gene encoding the Y-box protein DrC_00456, a homologue of DeY1 in Dugesia etrusa (Salvetti et al., 2002) and MSY4 in S. mediterranea (Wang et al., 2010), all of which are specific markers for the testes, was not detected by WISH anywhere in the body (Fig. 6G). Moreover, there seemed to be few oogonia in the ventral periphery of the ovary (Fig.6A). Meanwhile, the somatic reproductive organs (copulatory apparatus and yolk glands) were morphologically normal (Fig. 6 B,C).

Furthermore, after being maintained with RNAi food for a month following the RNAi sexualization, the Dr-nanos(RNAi)-sexualized worms that lacked testes were maintained for 5 months and fed their usual food (chicken liver without double strand RNA(dsRNA)). Most individuals returned to morphologically asexual reproduction involving repeated fission and regeneration. Histological observations revealed that the copulatory apparatus had disappeared, the morphological ovary no longer existed, and instead, only the oogonia or stem cells were observed.

\section{Discussion}

\section{Germline stem cells express nanos in D. ryukyuensis}

Nanos proteins contain 2 conserved NANOS zinc finger motifs, which are known to be necessary for the formation of germ cells 
and for abdominal segmentation in Drosophila. It is reported that the C-terminal region following these motifs, which is conserved in insects, is required for abdominal segmentation and the migration of the PGCs to the gonads during embryogenesis but is not required for germ cell formation (Arrizabalaga and Lehmann, 1999). In this study, a nanos ortholog was identified in $D$. ryukyuensis as Dr-nanos, which encoded a protein with 2 conserved NANOS zinc finger motifs. However, the C-terminal domain in NANOS did not exist in Dr-nanos, as observed in 2 nanos genes identified in different planarians (Sato et al., 2006; Wang et al., 2007; HandbergThorsager and Saló, 2007). It is suggested that the function of nanos in planarians is limited to the formation of germ cells.

In asexual worms, a functional ovary does not exist. Instead, GSCs are formed and maintained in the prospective ovarian region and may contribute to switching between the reproductive modes in these worms. Dr-nanos was expressed in the early oogonia and spermatogonia of the sexualized worms and in the oogonia-like cells of the primordial ovaries of the asexual worms. Furthermore, Dr-nanos expression signals were detected in the cells of the presumed ovaries, whereas they were not detected in the prospective testicular region. In 2 other planarian species, $D$. japonica and $S$. mediterranea, nanos ortholog genes were found to be Djnos and Smed-nanos, respectively. The expression signals of Djnos and Smed-nanos in the asexual worms were distributed in the prospective testes and ovarian regions (Sato et al., 2006; Wang etal., 2007; Handberg-Thorsager and Saló, 2007). The male GSCs in the asexual form of $D$. ryukyuensis may be non-existent or too small to be observed, and it is expected that the GSCs or spermatogonia (without GSC formation) develop from the neoblasts at the beginning of the sexualization process.

\section{Dr-nanos function during germline development}

The results of the Dr-nanos knockdown in the mature sexualized worms, along with the expression pattern of Dr-nanos, indicated that Dr-nanos is necessary for the formation and the maintenance of the oogonia, spermatogonia, and GSCs. Additionally, it appears that Dr-nanos is not involved in the later process of gametogenesis because the oocytes, spermatids, and sperm were present, even when the cells were eliminated before meiosis. The results of the Dr-nanos knockdown during the sexualization process showed that the spermatogonia and the cells at subsequent developmental stages did not appear, and that the testes had not formed at all. This supports the possibility that Dr-nanos is essential during the early stages of germline specification in the testes. Meanwhile, the ovaries were not eliminated completely, and the oogonia had decreased. This may be because GSCs are present only in the presumed ovarian region, which is developed by sexualization, in asexual worms. However, after a certain time, the oogonia may not be formed, as the replenishment of the GSC supply (formed from the neoblasts) or the GSC turnover by self-renewal does not occur. Moreover, the suppression of Dr-nanos expression did not influence the formation of the somatic reproductive organs, which showed that $D r$-nanos has a specialized key role in germline development.

Incidentally, the sexualized Dr-nanos(RNAi) worms, which lack testes, induced the regression of the ovaries and the copulatory apparatus and became asexually reproductive after discontinuation of the gene silencing (data not shown). Ovary formation occurs earlier than testes formation during the sexualization process. Most likely, the formation of the ovary is induced by signals at the beginning of the sexualization process, but the maintenance of the ovaries, copulatory apparatus, and yolk glands (i.e., maintenance of the sexual state) might depend on the testes.

\section{Comparison of germline stem cell (GSC) regulation between planarian species}

In the asexual worm, the expression of Dr-nanos was only observed in the ovarian primordia; however, in $D$. japonica and $S$. mediterranea, nanos mRNA was expressed not only in the ovarian primordia, but also in the prospective testes region (Sato et al., 2006; Wang et al., 2007; Handberg-Thorsager and Saló, 2007). Their phenotypes in the sexual worm after RNAi treatment differed from the phenotype of $D$. ryukyuensis, which
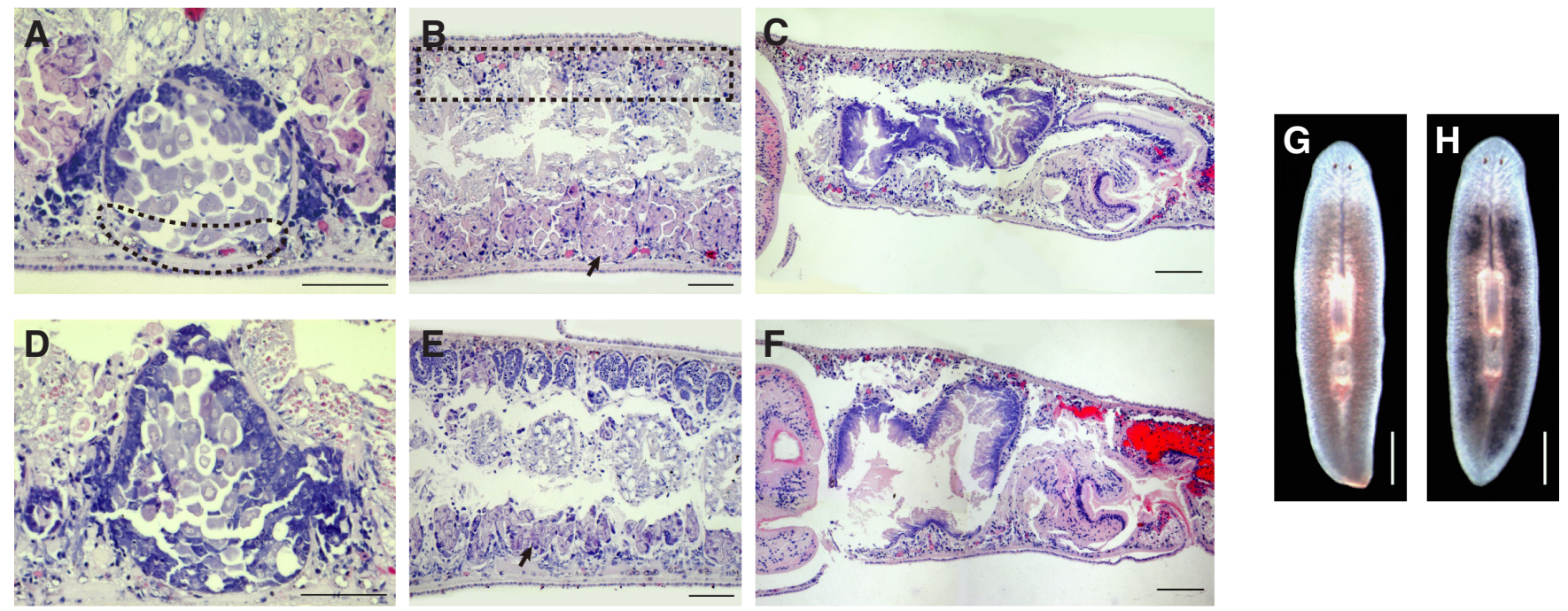

Fig. 6. Analysis of Dr-nanos function during sexualization. (A-F) HE staining of sagittal sections of the sexualizing worms at 40 days with Dr-nanos RNAi. (A-C) Dr-nanos(RNAi) worm and (D-F) EGFP(RNAi) control worm. (A, D) Ovaries; (B, E) region around the testes; and (C, F) copulatory apparatus. Arrows indicate yolk glands. The affected regions are enclosed with a dotted line. Scale bars, 100 um. (G,H) WISH for DrC_00456, a specific marker for the testes. (G) Dr-nanos(RNAi) worm and (H) EGFP(RNAi) control worm. Scale bars, $1 \mathrm{~mm}$. 
lacked testes and had abnormal ovaries. All the reproductive organs - the ovaries, testes, and copulatory apparatus-were not developed in the Smed-nanos(RNAi) sexual worms, whereas no special phenotype was observed during the sexualization process in the Djnos(RNAi) worms. In a comparison of reproductive abilities, both $D$. ryukyuensis and $D$. japonica can switch their reproductive modes, but $S$. mediterranea cannot exercise experimental sexualization. Furthermore, D. ryukyuensis $\mathrm{OH}$ strains change from the asexual to sexual reproductive modes more easily and promptly than $D$. japonica. Therefore, the planarian system for germ cell specification such as the spatial and temporal regulation of the GSCs differs among planarian species. From these results, it could be demonstrated that the asexual worm form of $D$. ryukyuensis contains only ovarian GSCs and retains the asexual reproductive state more than $D$. japonica and $S$. mediterranea, which instead have both ovarian and testicular GSCs. Therefore, differences in their nanos gene expression patterns and phenotypes of nanos RNAi would be caused by the GSC condition in each planarian species. Among them, we considered the nanos functions in the early stages of germ cell specification, which are common to the planarian species. The molecular mechanism controlling NANOS has not yet been fully clarified, except for in Drosophila; therefore, our sexualization experiment carried out on $D$. ryukyuensis is a powerful tool to study the development of neoblasts into testicular GSCs. This research will provide further understanding of NANOS in the metazoans.

Dr-nanos, the nanos homolog in D. ryukyuensis is expressed in the presumed GSCs and immature germline cells. However, the expression of nanos-positive GSCs and the phenotypes resulting from the knockdown of nanos are differ according to the species and may depend on the difference in time points when the GSCs are regulated, the germ cell formation process, and reproductive mode of each species. In the asexual worm form of D. ryukyuensis, only the ovarian GSCs and not the testicular GSCs are specified; therefore, the cell development can be observed during the specification process as the neoblasts convert to testicular GSCs during $D$. ryukyuensis sexualization experiments. In order to study the mechanisms underlying the germ cell developmental process in $D$. ryukyuensis, further studies must be conducted to clarify the regulation of the nanosgene and the function of the NANOS protein.

\section{Materials and Methods}

\section{Animals}

An exclusively fissiparous strain, the $\mathrm{OH}$ strain of the planarian $D$. ryukyuensis, was provided by Dr. S. Ishida of Hirosaki University, Hirosaki, Japan. The planarians were maintained at $20^{\circ} \mathrm{C}$ in dechlorinated tap water and were fed chicken liver. The $\mathrm{OH}$ strain was completely sexualized using the feeding procedure described by Kobayashi et al., (1999).

\section{Identification and cloning of Dr-nanos genes}

Clones of the nanos homolog, Dr_sW_003_K04 and Dr_sW_028_K12 in the $D$. ryukyuensis ESTs (Ishizuka et al., 2007), included the complete $D r$-nanos open reading frame. The full-length sequences of $D r$-nanos were obtained using $3^{\prime}$ and $5^{\prime}$ rapid amplification of CDNA ends (RACE) using a

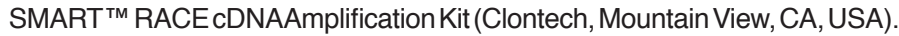

\section{In situ hybridization and histological analysis}

WISH was carried out as described previously (Umesono et al., 1997; Higuchi etal., 2008). Digoxigenin-labeled riboprobes were synthesized using an in vitro Transcription Kit (Roche Applied Science, Indianapolis, IN, USA).
In situ hybridization of tissue sections was performed as previously described by Koboyashi et al.,1998); the fixative and the hybridization buffer were modified as below. Recipients were treated with $2 \%(\mathrm{v} / \mathrm{v})$ hydrochloric acid in 20 mM HEPES(4-(2-hydroxyethyl)-1-piperazineethanesulfonic acid))buffered $5 / 8$ Holtfreter's solution $(\mathrm{pH} 7.4)$ for $5 \mathrm{~min}$ at $4^{\circ} \mathrm{C}$, which was then replaced with fixative (4\% paraformaldehyde in $20 \mathrm{mM}$ HEPES-buffered $5 / 8$ Holtfreter's solution, $\mathrm{pH} 7.4$ ) for $12-16 \mathrm{~h}$, at $4^{\circ} \mathrm{C}$. Fixed samples were dehydrated gradually through an ethanol series, clearZed with xylene, and embedded in Paraplast ${ }^{\mathrm{TM}}$ Plus (Sherwood Medical, St. Louis, MO, USA). Transverse and sagittal sections were cut at a thickness of $4 \mu \mathrm{m}$. Sections were used for in situ hybridization or stained with hematoxylin and eosin (HE). Sexualized worms were fully matured by feeding with chicken liver several times, after complete sexualization, using the feeding procedure described by Kobayashi et al., (1999).

\section{$X$-ray irradiation}

The planarians were placed on wet filter paper on ice and exposed to $10 \mathrm{~Gy}$ of $\mathrm{X}$-ray irradiation using an ultrasoft $\mathrm{X}$-ray generator SOFTEX E-3 (Softex Co., Ebina, Japan).

\section{Reverse transcription polymerase chain reaction (RT-PCR)}

Total RNA was isolated using Sepasol (Nacalai Tesque, Kyoto, Japan), treated with DNase (Promega Corp., Madison, WI, USA), and transcribed using a ReverTra Ace kit (Toyobo, Tokyo, Japan). RT-PCR for the GAPDH gene was also performed as an internal amplification control. Drpcna is a homolog of Djpcna, which is present in proliferative- and X-ray-sensitive cells that correspond to the neoblasts (Orii et al., 2005). PCR amplification of cDNA was performed using a set of specific oligonucleotide primers:

Dr-nanos

Forward: 5'-GACAAATATGAATCCTTCACTT-3'

Reverse: 5'-GAATTTGAAGAAATTGGACAATA-3'

DrGAPDH

Forward: 5'-AGTCGATGGTCATGCAATGTCTGT-3'

Reverse: 5'-TAATATTCTGAGCTGCACCTCTGCC-3' Drpcna

Forward, 5'-CCTCGGAAGTAACGACTCTC-3'

Reverse, 5'-ATAGCCTCACCCATCTGACT-3'.

\section{RNAi gene silencing}

Fragments of Dr-nanos were amplified by PCR and were cloned into pGEM ${ }^{\circledR}$ T-vectors (Promega Corp., Madison, WI, USA). The following primers were used:

Dr-nanos dsRNA

forward, 5'-GCTACGAGCTCACTTTGGCAATCGGTAAAC-3' reverse, 5'-AGTTCAGGTACCTTGATTGTATGAGCAAAATCACC-3'.

Two templates for dsRNA synthesis that added a T7 promoter to one end of each were amplified by PCR using the plasmid described above using 2 sets of primers. dsRNAs were synthesized from each template using T7 RiboMAX ${ }^{\mathrm{TM}}$ (Promega Corp., Madison, WI, USA).

dsRNA template-1

forward, 5'-GTAAAACGACGGCCAGT-3';

reverse, 5'-CTCAAGCTATGCATCCAACG-3'; dsRNA template-2

forward, 5'-ATTGGGCCCGACGTCGCATG-3'; reverse, 5'-GCGTAATACTACTCACTATAGGGCTCAAGCTATG CATCCAACGCG-3'.

For 10 animals, $4-10 \mu \mathrm{g} / 10 \mu \mathrm{l}$ dsRNA was mixed with $20 \mu \mathrm{l} 70 \%$ liver, $7.5 \mu \mathrm{l} 2 \%$ ultra-low gelling temperature agarose, and red food coloring, and allowed to solidify on ice.

To silence the nanos function and to maintain homeostasis in the sexualized worms, RNAi food was fed to the mature sexualized worms every 3 days, and the worms were subsequently maintained for 50 days. Two animals were fixed every 10 days, and were observed histologically. Control animals were fed RNAi food with enhanced green 
fluorescent protein (EGFP) dsRNA, which is not found in planarians.

To silence the nanos function during sexualization, RNAi food was fed to the asexual worms twice a day every 3 days. After 5 days, minced Bdellocephala brunnea was fed to the worms every day for 40 days, and $20 \mu \mathrm{g}$ dsRNA was mixed with $B$. brunnea twice a week. Four animals were fixed every 10 days, and were observed histologically. After RNAi treatment during the sexualization process, planarians were maintained on their usual food (chicken liver).

RT-PCR was performed to confirm gene suppression using the primers described above. These primers were designed to contain the region used in the RNAi.

\section{Acknowledgments}

We would like to thank Dr. Sachiko Ishida, Hirosaki University, for his gift of the $\mathrm{OH}$ strain. We also thank Dr. Phil Newmark, for advice and discussions concerning the expression of nanos and its function between planarian species. This work was supported in part by grants from the Ministry of Education, Culture, Sports, Science, and Technology awarded to M.M (16086209), and from the Keio Leading-edge Laboratory of Science \& Technology Research Grant for the Ph.D. Program awarded to H.N.

\section{References}

ARRIZABALAGA, G. and LEHMANN, R. (1999). A selective screen reveals discrete functional domains in Drosophila Nanos. Genetics 153: 1825-1838.

BAGUÑÀ, J., SALÓ, E. and AULADELL, C. (1989). Regeneration and pattern formation in planarians. III. Evidence that neoblasts are totipotent stem cells and the cells. Development 107: 77-86.

EXTAVOUR, C.G. and AKAM, M. (2003). Mechanisms of germ cell specification across the metazoans: epigenesis and preformation. Development 130: 5869-5884

FORBES, A. and LEHMANN, R. (1998). Nanos and Pumilio have critical roles in the development and function of Drosophila germline stem cells. Development 125: 679-690.

HANDBERG-THORSAGER, M. and SALÓ, E. (2007). The planarian nanos-like gene Smednos is expressed in germline and eye precursor cells during development and regeneration. Dev Genes Evol 217: 403-411.

HAYASHI, Y., HAYASHI, M. and KOBAYASHI, S. (2004). Nanos suppresses somatic cell fate in Drosophila germ line. Proc Nat Acad Sci USA 101: 10338-10342.

HIGUCHI, S., HAYASHI, T., TARUI, H., NISHIMURA, O., NISHIMURA, K., SHIBATA, N., SAKAMOTO, H. and AGATA, K. (2008). Expression and functional analysis of musashi-like genes in planarian CNS regeneration. Mech Dev 125: 631-645.

ISHIZUKA, H., MAEZAWA, T., KAWAUCHI, J., NODONO, H., HIRAO, Y., NISHIMURA, O., NAKAGAWA, H. SEKII, K., TASAKA, K., TARUI, H., AGATA, K., HOSHI, M., KOBAYASHI, K., SAKAKIBAYA, Y. and MATSUMOTO, M. (2007). The Dugesia ryukyuensis Database as a Molecular Resource for Studying Switching of the Reproductive System. Zool Sci 24: 31-37.

JENKINS, M.M. (1967). Aspects of planarian biology and behavior, in: Corning, W.C., Ratner, S.C. (Eds.), Chemistry of Learning Plenum Press, New York, pp. 117-143.

JOHNSON, A.D., DRUM, M., BACHVAROVA, R.F., MASI, T., WHITE, M.E. and CROTHER, B.I. (2003). Evolution of predetermined germ cells in vertebrate embryos: implications for macroevolution. Evol Dev 5: 414-431.

KOBAYASHI, C., KOBAYASHI, S., ORII, H., WATANABE, K. and AGATA, K. (1998). Identification of Two Distinct Muscles in the Planarian Dugesia japonica by their Expression of Myosin Heavy Chain Genes. Zool Sci 15: 861-869.

KOBAYASHI, K., KOYANAGI, R., MATSUMOTO, M., CABRERA, J.P. and HOSHI, M. (1999). Switching from Asexual to Sexual Reproduction in the Planarian Dugesia ryukyuensis: Bioassay System and Basic Description of Sexualizing Process. Zool Sci 16: 291-298.

KOBAYASHO, K. and MOTONORI, HOSHI. (2011). Sex-inducing effect of a hydrophilic fraction on reproductive switching in the planarian Dugesia ryukyuensis (Seriata, Tricladida). Front Zool 8: 23.

KOBAYASHI, S, YAMADA, M., ASAOKA, M. and KITAMURA, T. (1996). Essential role of the posterior morphogen nanos for germline development in Drosophila. Nature 380: 708-711.

KÖPRUNNER, M., THISSE, C., THISSE, B. and RAZ, E. (2001). A zebrafish nanosrelated gene is essential for the development of primordial germ cells. Genes Dev 15: 2877-2885.

LEHMANN, R. and NÜSSLEIN-VOLHARD, C. (1991). The maternal gene nanos has a central role in posterior pattern formation of the Drosophila embryo. Develop ment 112: 679-691.

LIN, H. (1997). The tao of stem cells in the germline. Annu Rev Genet 31: 455-491.

LIN, H. (1998). The self-renewing mechanism of stem cells in the germline. Curr Opin Cell Biol 10: 687-693.

MORGAN, T.H. (1901). Growth and regeneration in Planaria lugubris. Arch Ent mechder Org 13: 179-212.

NEWMARK, P.A., WANG, Y. and CHONG, T. (2008). Germ cell specification and regeneration in planarians. Cold Spring Harb Symp Quant Biol 73: 573-581.

ORII, H., SAKURAI, T. and WATANABE, K. (2005). Distribution of the stem cells (neoblasts) in the planarian Dugesia japonica. Dev Genes Evol 215: 143-157.

PARISI, M. and LIN, H. (2000). Translational repression: a duet of Nanos and Pumilio. Curr Biol 10: R81-83.

SAKURAI, T. (1981). Sexual induction by feeding in an asexual strain of the freshwater planarian, Dugesia japonica. Annot Zool Jap 54: 103-112.

SALVETTI, A., LENA, A., ROSSI, L., DERI, P., CECCHETTINI, A., BASISTONI, R. and GREMIGNI, A. (2002). Characterization of DeY1, a novel Y-box gene specifically expressed in differentiating male germ cells of planarians. Gene Expr. Patterns 2: 195-200.

SATO, K., SHIBATA, N., ORII, H., AMIKURA, R., SAKURAI, T., AGATA, K. KOBAYASHI, $S$. and WATANABE, K. (2006). Identification and origin of the germline stem cells as revealed by the expression of nanos-related gene in planarians. Dev Growth Differ 48: 615- 628.

SUBRAMANIAM, K. and SEYDOUX, G. (1999). nos-1 and nos-2, two genes related to Drosophila nanos, regulate primordial germ cell development and survival in Caenorhabditis elegans. Development 126: 4861-4871.

SUZUKI, A., TSUDA, M. and SAGA, Y. (2007). Functional redundancy among Nanos proteins and a distinct role of Nanos2 during male germ cell development. Development 134: 77-83.

TSUDA, M., SASAOKA, Y., KISO, M., ABE, K., HARAGUCHI, S., KOBAYASHI, S. and SAGA, Y. (2003). Conserved role of nanos proteins in germ cell development Science 301: 1239-1241.

UMESONO, Y., WATANABE, K. and AGATA, K. (1997). A planarian orthopedia homolog is specifically expressed in the branch region of both the mature and regenerating brain. Dev Growth Differ 39: 723-727.

WANG, C. and LEHMANN, R. (1991). Nanos is the localized posterior determinant in Drosophila. Cell 66: 637-647.

WANG, Y, ZAYAS, R. M., GUO, T. and NEWMARK, P.A. (2007). Nanos Function is Essential for Development and Regeneration of Planarian Germ Cells. Proc Natl Acad Sci USA 104: 5901-5906.

WANG, Y, STARY, J.M., WILHELM, J.E. and NEWMARK, P.A. (2010). A functional genomic screen in planarians identifies novel regulators of germ cell development. Genes Dev 24: 2081-2092.

WOLFF, E. and DUBOIS, F. (1948). Sur la migration des cellules de regeneration chez les planaires. Rev Suisse Zool 55: 218-227. 


\section{Further Related Reading, published previously in the Int. J. Dev. Biol.}

Diverse miRNA spatial expression patterns suggest important roles in homeostasis and regeneration in planarians Cristina González-Estévez, Varvara Arseni, Roshana S. Thambyrajah, Daniel A. Felix and A. Aziz Aboobaker Int. J. Dev. Biol. (2009) 53: 493-505

Planarian regeneration: achievements and future directions after 20 years of research Emili Saló, Josep F. Abril, Teresa Adell, Francesc Cebriá, Kay Eckelt, Enrique Fernández-Taboada, Mette Handberg-Thorsager, Marta Iglesias, M Dolores Molina and Gustavo Rodríguez-Esteban

Int. J. Dev. Biol. (2009) 53: 1317-1327

Two msh/msx-related genes, Djmsh1 and Djmsh2, contribute to the early blastema growth during planarian head regeneration Linda Mannini, Paolo Deri, Vittorio Gremigni, Leonardo Rossi, Alessandra Salvetti and Renata Batistoni Int. J. Dev. Biol. (2008) 52: 943-952

From Planarians to Mammals - the many faces of regeneration Jerzy Moraczewski, Karolina Archacka, Edyta Brzoska, Maria-Anna Ciemerych, Iwona Grabowska, Katarzyna Janczyk-Ilach, Wladyslawa Streminska and Malgorzata Zimowska Int. J. Dev. Biol. (2008) 52: 219-227

Expression of a retinal homeobox $(\mathbf{R x})$ gene during planarian regeneration Linda Mannini, Paolo Deri, Jacopo Picchi and Renata Batistoni Int. J. Dev. Biol. (2008) 52: 1113-1117

Characterization of novel genes expressed specifically in the sexual organs of the planarian Dugesia ryukyuensis

Sumitaka Hase, Emiko Kashiwagi, Kazuya Kobayashi, Motonori Hoshi and Midori Matsumoto

Int. J. Dev. Biol. (2007) 51: 345-349

Expression of DjXnp, a novel member of the SNF2-like ATP-dependent chromatin remodelling genes, in intact and regenerating planarians. Leonardo Rossi, Paolo Deri, Ilaria Andreoli, Vittorio Gremigni, Alessandra Salvetti and Renata Batistoni Int. J. Dev. Biol. (2003) 47: 293-298

The genetic control of eye development and its implications for the evolution of the various eye-types.

Walter J Gehring Int. J. Dev. Biol. (2002) 46: 65-73

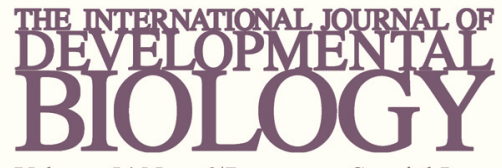

Volume 54 Nos. 6/7
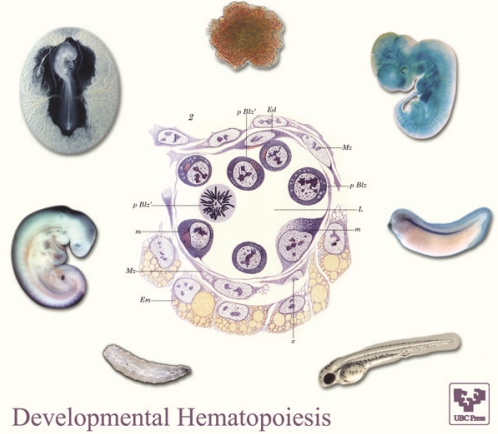

5 yr ISI Impact Factor $(2010)=2.961$

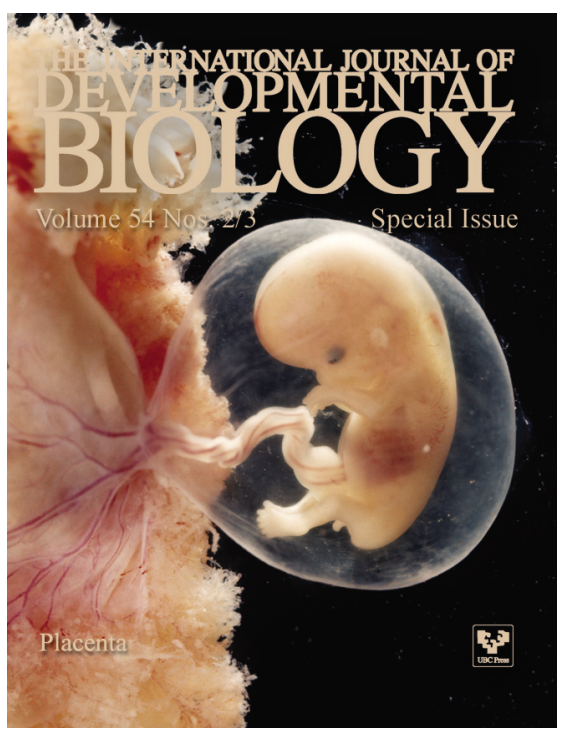

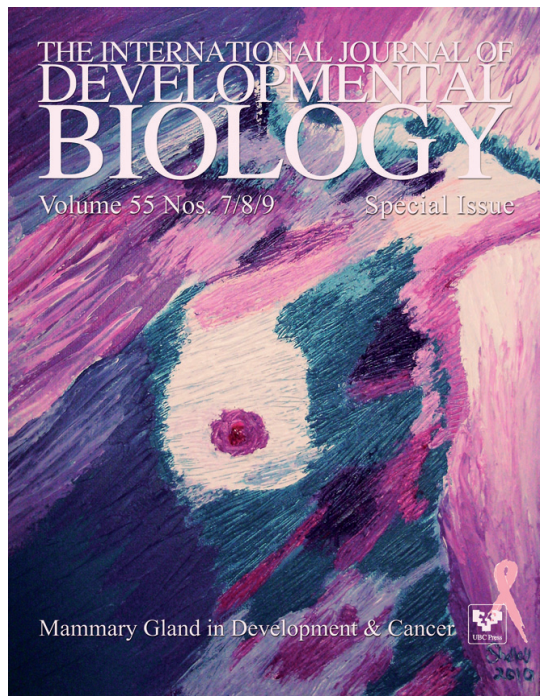

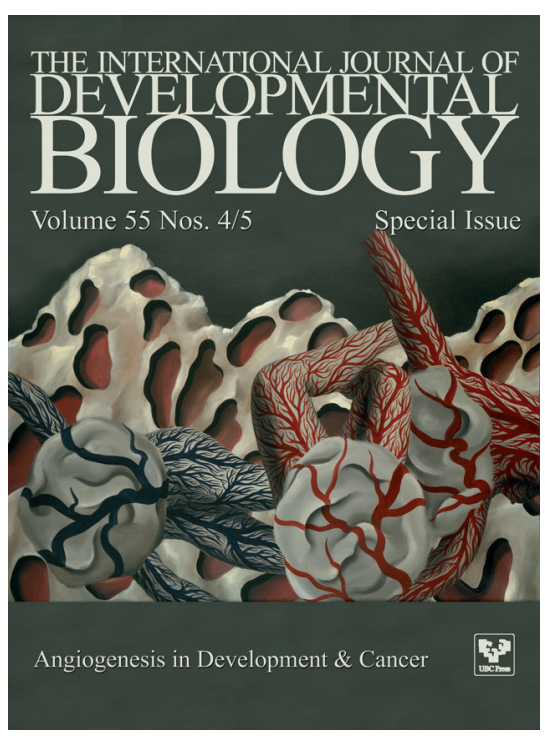

